\title{
Intra-molecular Conformational Stability in Human Growth Hormone
}

\author{
R. P. Koirala, B. Thapa, S. P. Khanal, \\ R. P. Adhikari and N. P. Adhikari
}

Journal of Nepal Physical Society

Volume 6, Issue 2, December 2020

ISSN : 2392-473X (Print), 2738-9537 (Online)

Editors:

Dr. Binod Adhikari

Dr. Bhawani Joshi

Dr. Manoj Kumar Yadav

Dr. Krishna Rai

Dr. Rajendra Prasad Adhikari

Mr. Kiran Pudasainee

JNPS, 6 (2), 41-49 (2020)

DOI: http://doi.org/10.3126/jnphyssoc.v6i2.34856

Published by:

Nepal Physical Society

P.O. Box: 2934

Tri-Chandra Campus

Kathmandu, Nepal

Email: npseditor@gmail.com

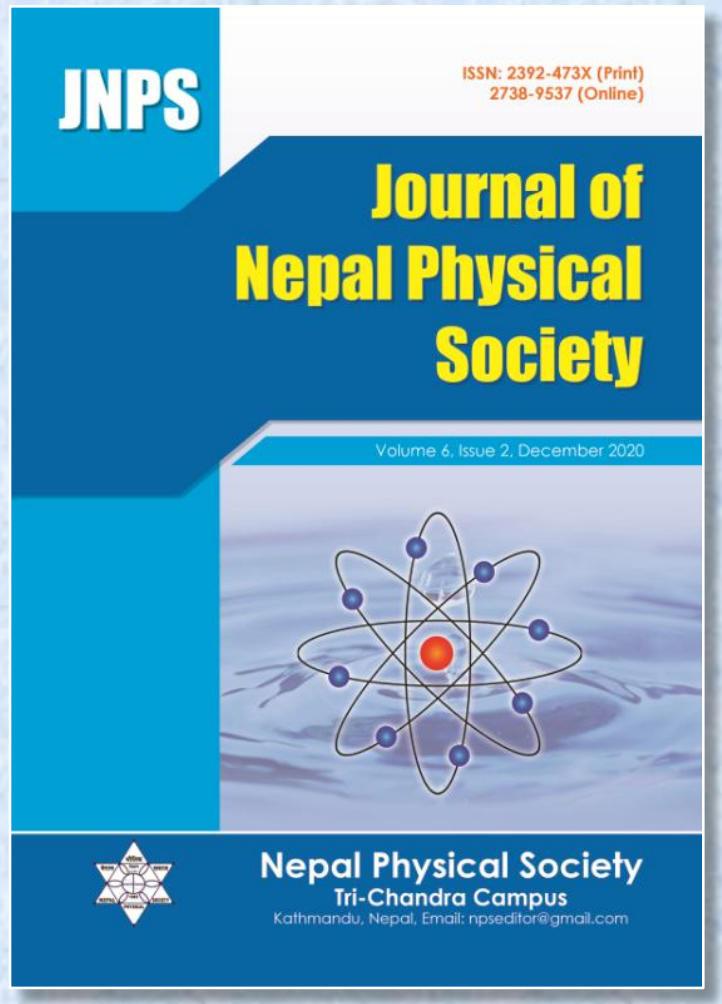




\title{
Intra-molecular Conformational Stability in Human Growth Hormone
}

\author{
R. P. Koirala ${ }^{1}$, B. Thapa ${ }^{2}$, S. P. Khanal ${ }^{1}$, R. P. Adhikari ${ }^{3}$ and N. P. Adhikari ${ }^{1 \text {,* }}$ \\ ${ }^{1}$ Central Department of Physics, Tribhuvan University, Kathmandu, Nepal \\ ${ }^{2}$ Padma Kanya Multiple Campus, Tribhuvan University, Kathmandu, Nepal \\ ${ }^{3}$ Department of Natural Sciences, Kathmandu University, Dhulikhel, Nepal \\ *Corresponding Email: narayan.adhikari@cdp.tu.edu.np
}

Received: 22 October, 2020; Revised: 28 November, 2020; Accepted: 25 December, 2020

\begin{abstract}
Human growth hormone (hGH) is synthesized, stored and secreted by somatotropic cells within the lateral wings of the anterior lobe of pituitary glands; and is transported to other organs of human body. Study of intra-molecular structure and its binding mechanisms within the molecule gives more insight of structural stability of the molecule and is also essential in drug designing. In this article, we have investigated the various bonded and non-bonded interactions that contribute for the conformation of entire structure of the hGH molecule using molecular dynamics (MD) simulation. The MD outcomes show that the molecule is hydrophobic in nature. In its conformation, several types of interactions exist, such as disulphide bridges (bonded) and nonbonded: hydrogen bond, hydrophobic, aromatic-aromatic, ionic, aromatic-sulphur, cation-pi.
\end{abstract}

Keywords: Intra-binding, Growth hormone, Hydrophobic, Aromatic.

\section{INTRODUCTION}

Personal height is the highly concerned matter for everyone. It is also a measure of health conditions [1]. Human growth hormone (hGH) is essential for the proper development of height as well as every part of body. It is a single chain peptide hormone that stimulates growth, reproduction and regeneration of cell in human [2]. Although growth hormone is found in other animals, its effect is significant only in human and old world monkey. Many researches have revealed that the variation of amino acid sequence of $\mathrm{hGH}$ is significant in different species [3]. Even though the sequence is different, its role in other animals is also similar to that in human body. This hormone contains 191 amino acid residues with 22,124 daltons molecular weight. It is synthesized, stored and secreted by somatotropic cells within the lateral wings of the anterior lobe of pituitary glands [4]. The hormone after secretion from the gland mixes into the blood stream and is transported into body cells [5]. The structure includes four helices necessary for the functional interaction with the growth hormone (GH) receptor [6]. Human growth hormone is essential in physical development. Main problem of human growth in childhood is the short stature, i.e., insufficient growth in accordance with age; and delayed sexual maturity in adulthood [7].

Human growth hormone, also known as somatotropin, provides important contribution in human development. It increases the concentration of glucose and free fatty acids [1]. It is legally prescribed as a drug to treat the children's growth disorders and adult growth hormone deficiency. Moreover, it is also used in raising livestock more efficiently in industrial agriculture.

Besides its functions of somatic and bone growth as well as increase in the size and mass of organs and tissues, hGH also influences in the functioning of proteins, carbohydrates and lipids metabolisms [8]. These mechanisms caused by hGH are basically due to its ability to bind with specific target cell receptor. hGH surface specifically favors for the inter-molecular bindings at the interfacial region of receptor. Inter-molecular binding occurs in the expense of intra-molecular binding, which ultimately lowers the conformation stability of the molecule $[9,10]$. There are several non-bonded 
interactions to form a macromolecule a stable structure. Hydrogen bonding play a pivotal role in the formation of secondary structure of a protein. Likewise, disulphide bond is a covalent bonding to give the shape in tertiary structure. Similarly, aromatic-aromatic interactions between two aromatic rings separated by distance $4.5 \AA$ to $7 \AA$ provide the conformation stability. This interaction is energetically favorable and basically applies to form the tertiary and quaternary structure $[11,12]$. Similarly, electrostatic and van der Waals interactions are pervasive in intra- and intermolecular bindings [13]. Likewise, hydrophobic interaction has influences in conformation changes of protein molecule in aqueous environment [14].

In this work, we have used molecular dynamics simulation to study the intra-molecular interactions and the contributions of hydrogen bonding and several other interactions such as hydrophobic, ionic, aromatic-aromatic, aromatic-sulphur and cation-pi that are responsible to provide the stability of hGH molecule. Moreover, we have compared the intra-molecular interactions of amino acid residues in static structure with the structure obtained from molecular dynamics simulations. To our best knowledge, the comprehensive study on molecular stability of this hormone has not been studied yet via MD run. We believe that this work aids the indepth knowledge about the binding of amino acid residues in order to form the stable structure of the hormone in cellular environment.

\section{MATERIALS AND METHODS}

To perform molecular dynamics simulation, one needs initial structure of molecule (pdb file) and force field parameters. In this section, we describe system setup of the hGH molecule, force field parameters used in the present work and simulation details.

System setup: The molecular structure of human growth hormone was taken from protein data bank with PDBID 1HGU.pdb [15]. In original file, two amino acids were missing at the $\mathrm{N}$-terminus and $\mathrm{C}$ terminus positions, which were filled with software program CHARMM-GUI [16]. This software program was also used to generate new protein structure file (psf) and pdb files. To mimic the cellular environment, the hormone molecule was solvated in TIP3P water in a cubical box of dimensions $84 \times 84 \times 84 \AA^{3}$. As TIP3P water model resembles real water closely, we chose this model in this work. Furthermore, the molecular system had originally 4 excess negative electronic charges, so the system was electrically neutralized by adding $4 \mathrm{Na}+$ ions.

Force fields: Force fields contain topology and parameter files. Topology files are used to generate psf files and parameter files contain the information about parameters of potential energy functions. In the present work, we used CHARMM36m [17] force field.

Molecular Dynamics Simulation: All-atom molecular dynamics (MD) simulations were carried out by using NAnoscale Molecular Dynamics (NAMD) [18] simulation package. The Particle Mesh Edward (PME) was used to treat the longrange interactions with a $12.0 \AA$ non-bonded cut off. The energy minimization was performed for 10,000 steps, using the conjugate gradient algorithm. Energy minimization run removes the unwanted hindrances between the atoms in the system. Since this run is performed in $0 \mathrm{~K}$ temperature, the system chooses the local minimum energy state [19, 20]. After energy minimization, the system was equilibrated at $310 \mathrm{~K}$ under the isothermalisochoric conditions for $10 \mathrm{~ns}$ with $1 \mathrm{fs}$ time step $[21,22]$. Then, the production run was propagated for $100 \mathrm{~ns}$ under NVT simulation run taking time step 2 fs by using Langevin dynamics with a damping constant of $1 \mathrm{ps}^{-1}$.

The NAMD energy plugin package available in Visual Molecular Dynamics (VMD) [23] was used to estimate structural stability of the hGH molecule in aqueous environment. Protein interaction calculator (PIC) [24] has been used to analyze the intra-molecular interactions. The study on intramolecular interactions depicts the detailed insights on folded state of the targeted molecule.

\section{RESULTS AND DISCUSSION}

This research work has been carried out by molecular dynamics simulation to study the amino acid arrangements, their folding mechanisms, the contributions of hydrogen bonding, and various other non-bonded interactions to form such a structure of human growth hormone in aqueous environment. The solvent accessible surface area and the energy profiles of bonded and non-bonded interactions have also been investigated to know the contact area and the stability of hGH in the aqueous environments.

Structural stability of human growth hormone: The pre-requisite of examining the structural stability of any biomolecular system is the estimation of root mean square deviation (RMSD). RMSD measures the structural stability of entire molecule in the given environment. We have taken 
the hGH molecule in aqueous environment in order to resemble the cellular condition. RMSD of the molecule has been calculated from VMD analysis tool "RMSD Trajectory Tool". The mathematical relation to estimate the RMSD is,

$$
R M S D=\sqrt{\frac{1}{N} \sum_{i=1}^{N}\left(r_{i}(t)-r_{i}^{r e f}\right)^{2}}
$$

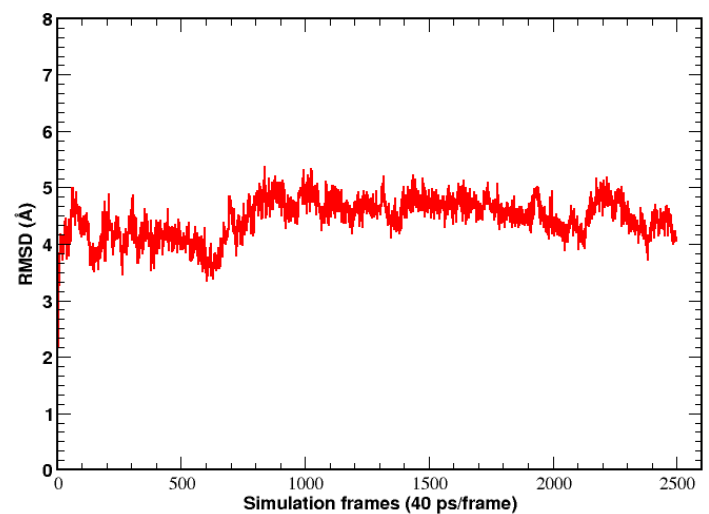

(i)
Where, $r_{i}(t)$ and $r_{i}^{\text {ref }}$ represent the current and reference coordinates of $i^{\text {th }}$ atom respectively and $\mathrm{N}$ is total number of atoms.

In this process, initial coordinates are taken as the reference and average deviation of molecule from the reference coordinates has been calculated in every frame of simulation. During the $100 \mathrm{~ns}$ NVT production run, the RMSD of the hGU was found fairly stable after 4 ns simulation (100 frames) time as shown in Fig. 1(i).

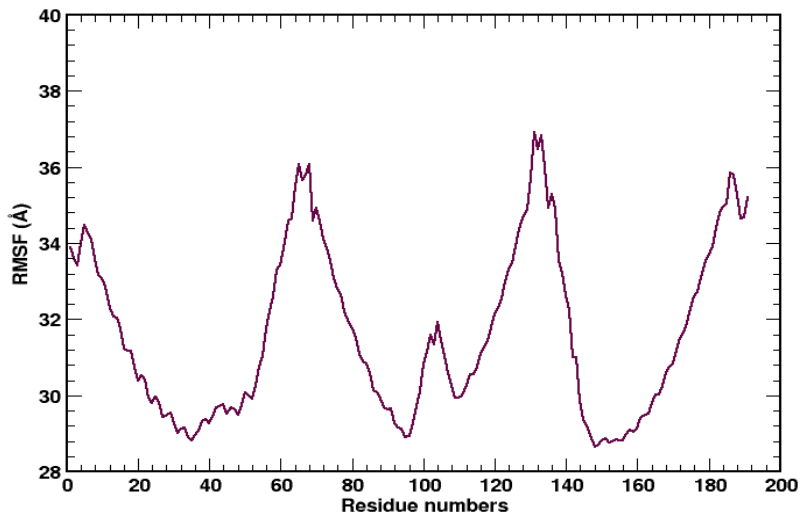

(ii)

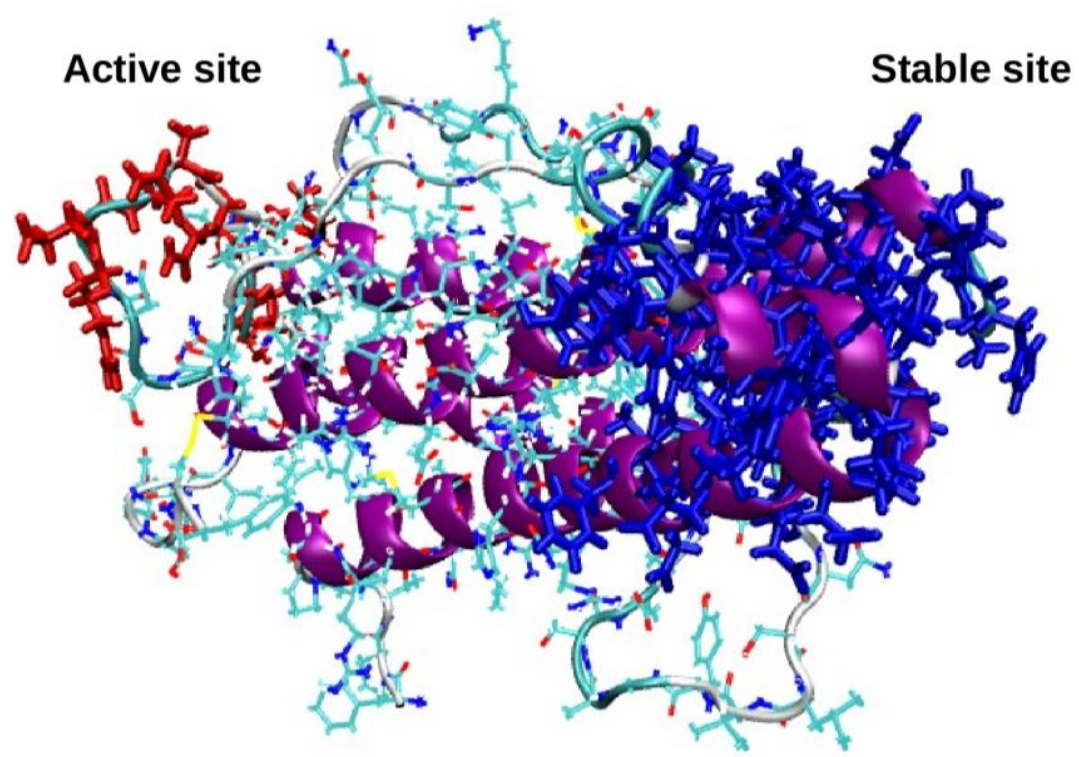

(iii)

Fig. 1: Structural characterization of hGH in aqueous environment (i) RMSD (ii) RMSF and (iii) hGH molecule.

In addition, we have also determined the fluctuation of alpha carbons of hGH residues during the simulations through root mean square fluctuation (RMSF) as shown in Fig. 1(ii), which gives the flexibility of residues within the molecular system. Greater value of RMSF of any alpha carbon implies the greater flexibility of corresponding amino acid in the protein chain and vice versa. The mathematical formula to find the RMSF is,

$$
R M S F=\sqrt{\left\langle r_{i}(t)-\left\langle r_{i}\right\rangle\right\rangle^{2}}
$$


where, $r_{i}(t)$ and $\left\langle r_{i}\right\rangle$ are the current position and average position respectively.

During the $100 \mathrm{~ns}$ simulation run, it has been observed that the regions of amino acid residues 64-68 and 130-136 are most fluctuating and the regions of 23-49, 88-98 and 144-164 are the most stable. The most fluctuating and stable regions are shown in Fig. 1 (iii).

Intra-binding potential energy: There are several interactions existing within a protein molecule to provide it a stable conformation. We have estimated the contributions of bonded and nonbonded potential energy in hGH molecule. Energy

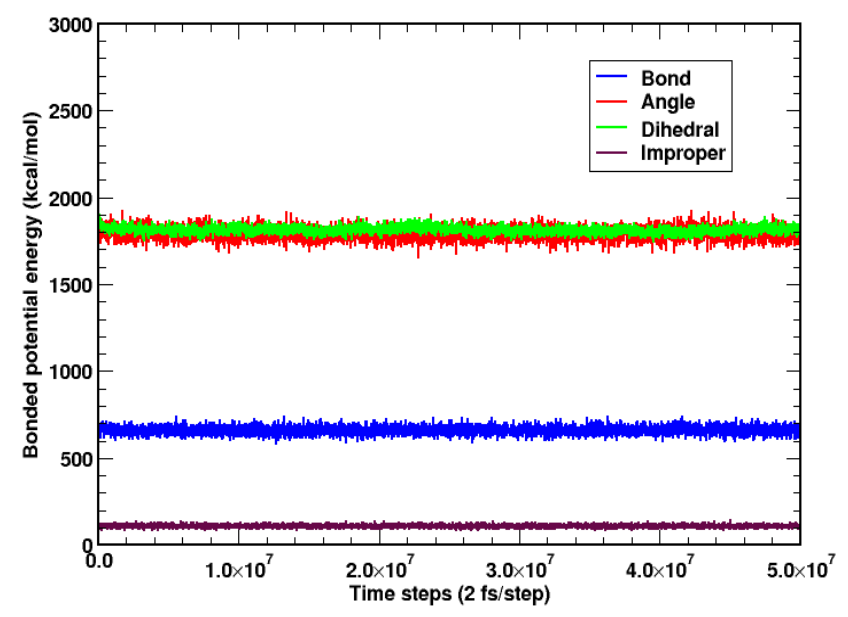

profile for bonded interactions have been determined from $100 \mathrm{~ns}$ simulation. From the energy profile, it has been observed that improper dihedral has lowest contributions; and the contributions of harmonic and dihedral angle show almost equal and the largest value as shown in Fig. 2. During the conformation of protein molecules, bonded energy provides the direct covalent link among the atoms, whereas the non-bonded energy contributes to fold the molecule forming the stable structure [25-27]. On analyzing the non-bonded energy, the contributions of electrostatic interaction has found to be significantly higher potential energy than that of van der Waals.

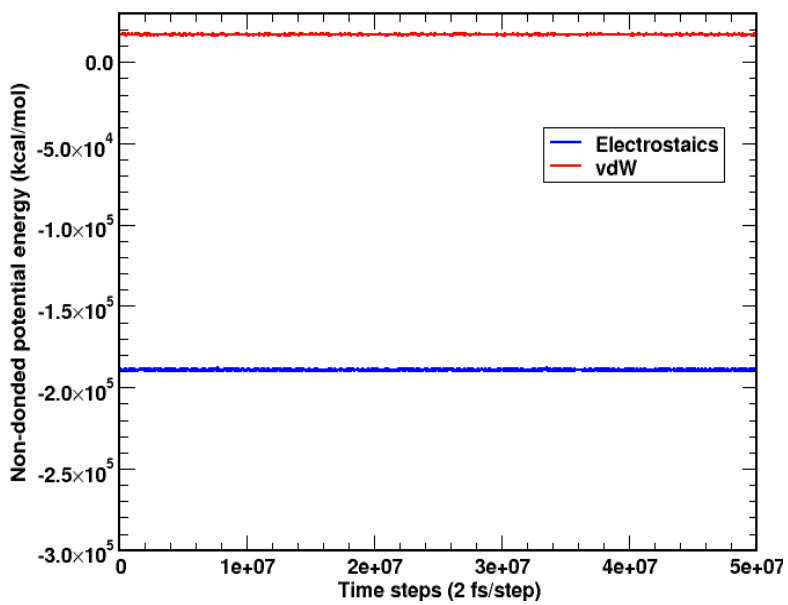

Fig. 2: Energy profiles for hGH (i) bonded (bond, angle, dihedral and improper) interaction (ii) non-bonded (electrostatics and van der Waals) interactions.

Intra-molecular hydrogen bonds: We have also investigated the hydrogen bonding within the molecule to form the hGH molecule. Hydrogen bonding is essential to form secondary structure of protein. The intra-molecular hydrogen bonding provides the important information for drug designing $[28,29]$. The number of hydrogen bonds can determine the conformational stability in the molecule. Its role in intermolecular interactions is also very important, which enhances the cooperativity among the molecules in proteinprotein, protein-ligand and protein-nucleic acid systems. We have found total 1294 hydrogen bonding in entire $100 \mathrm{~ns}$ simulation, and average of 65 hydrogen bonds persisted in each frame of simulation. The number of hydrogen bonds within the cutoff distance of $3.5 \AA$ has been presented in Fig. 3.

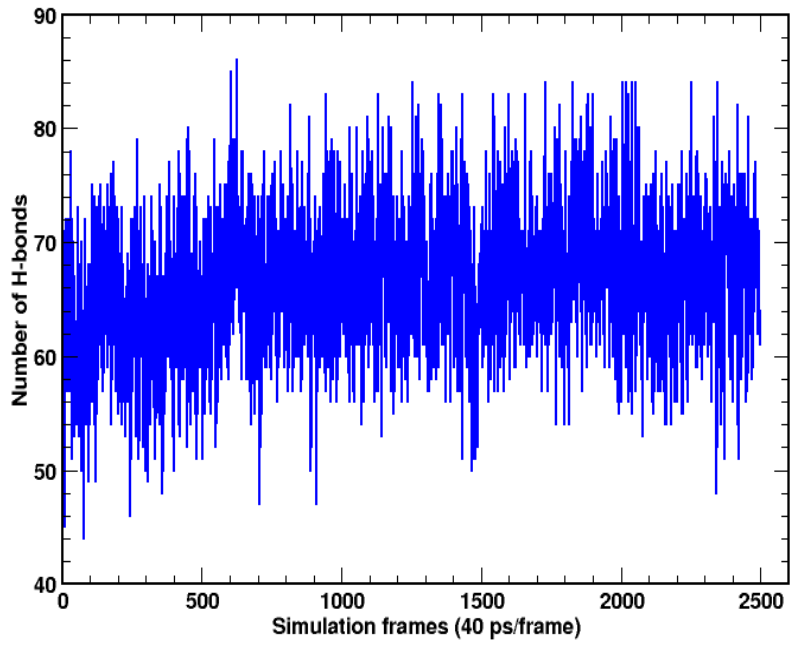

Fig. 3: Time variation of number of hydrogen bonding for intra-molecular binding in hGH. 
Many intra-molecular hydrogen bonds were observed in both static and dynamic conditions of the hGH structure. We have shown the hydrogen bonding patterns for three interacting pairs. These three pairs are the representative pairs for main chain - main chain, side chain - side chain and main chain - side chain as shown in Fig. 4. There are several such type of hydrogen bonds were observed to form the stable structure of the hormone.
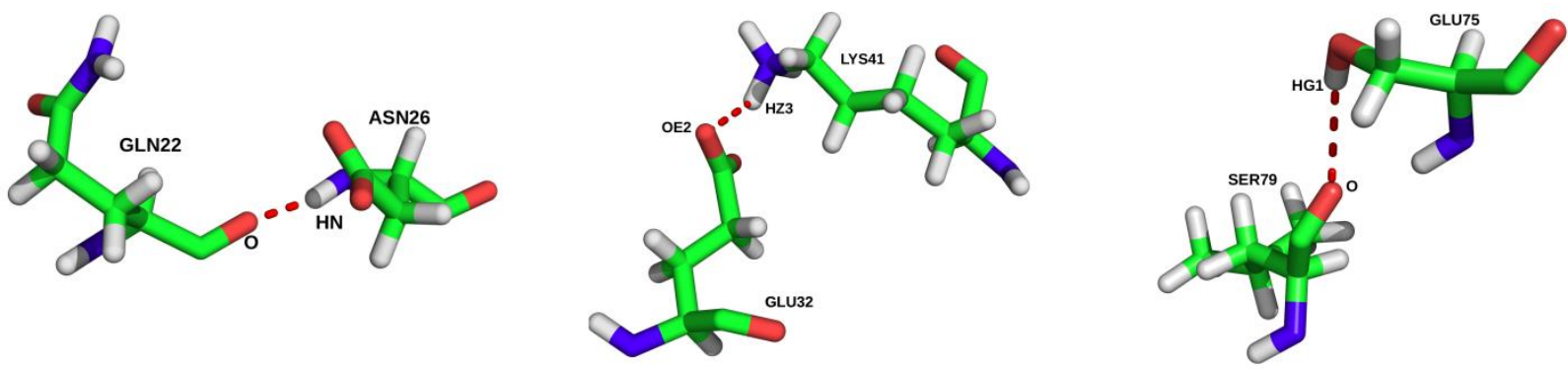

Fig. 4: Intra-molecular hydrogen bonds: main chain - main chain (left), side chain - side chain (middle) and main chain - side chain (right).

Surface accessible surface area (SASA): SASA measures the surface area of a molecule that contacts with the solvent molecules. We have taken water as the solvent to study how the amino acid residues residing on the surface of hGH behave in the aqueous environment. Fig. 5 shows the SASA plot with respect to simulation frame. During the 100 ns simulation, the overall value of SASA has been observed decreasing. The decreasing nature of graph shows the internal rearrangement of amino acid residues that resides on the surface. This shows that the molecule is hydrophobic in nature so that the surface residues tend to aggregate together to minimize the surface area exposed to water.

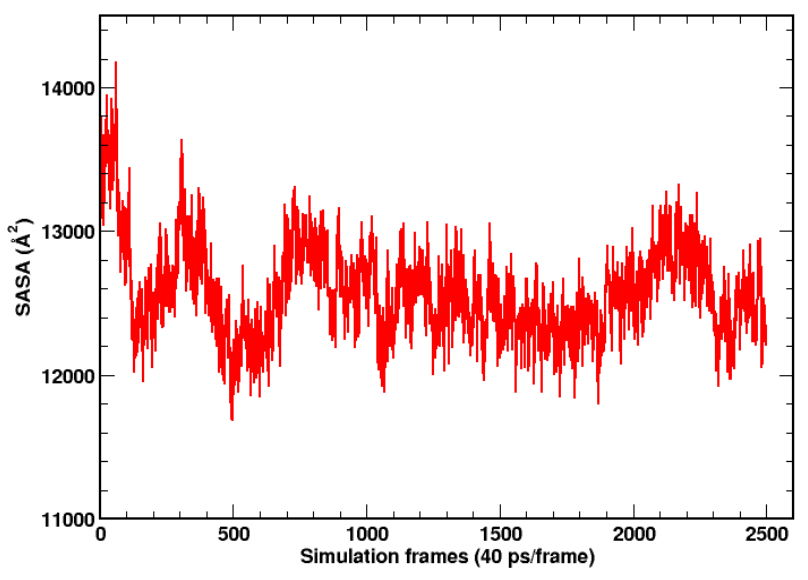

Fig. 5: Solvent accessible surface area (SASA) of hGH in water environment.

Intra-molecular Interactions: We have computed the various interactions present within the human growth hormone such as hydrophobic interactions, disulphide bridges, ionic interactions, aromaticaromatic interactions, aromatic-sulphur interactions and cation-pi interactions using protein interaction calculator (PIC) web server. We have compared these intra-molecular interactions in static structure with the most stable structure obtained from molecular dynamics simulations. The most stable structure was taken from the MD trajectories corresponding to the minimum SASA.

There are two disulphide bridges formed between two sulphur atoms of cysteine residues CYS182CYS189 and CYS53-CYS165 within $2.2 \AA$ in both structures. The distance between two cysteine residues in CYS182-CYS189 and CYS53-CYS165 is $2.02 \AA$ in static structure, whereas $2.06 \AA$ and $2.03 \AA$ respectively in the structure from dynamics.

The hydrophobic interactions are of particular importance for the structural stability of hGH. There are 116 interactions between the hydrophobic residues such as PHE, LEU, ALA, MET, PRO, VAL, ILE, TYR and TRP present in the static structure. We have observed the decrease in the solvent accessible surface area (SASA) of protein during the simulation in aqueous environment that suggests the hydrophobic nature of the molecule. This is corroborated further by the greater number of hydrophobic interactions in the dynamics, which are 143 as compared to 116 in static. The cut off distance of hydrophobic interaction was taken to be $5 \AA$.

In addition, the ionic interactions between the charged residues lying within the cut off distance of $6 \AA$ present in both structures of hGH protein are depicted in tables 1 and 2. We have observed 29 
ionic interactions in structure from dynamics, whereas only 11 in static. Salt bridges are the important interactions in forming the tertiary structure of protein molecules. They are the ionic interactions between charged atoms in residues lying within hydrogen bond distance $(3.5 \AA)$. In the structure obtained from MD simulation 7 saltbridges has been observed whereas only 2 saltbridges are obtained in static structure.

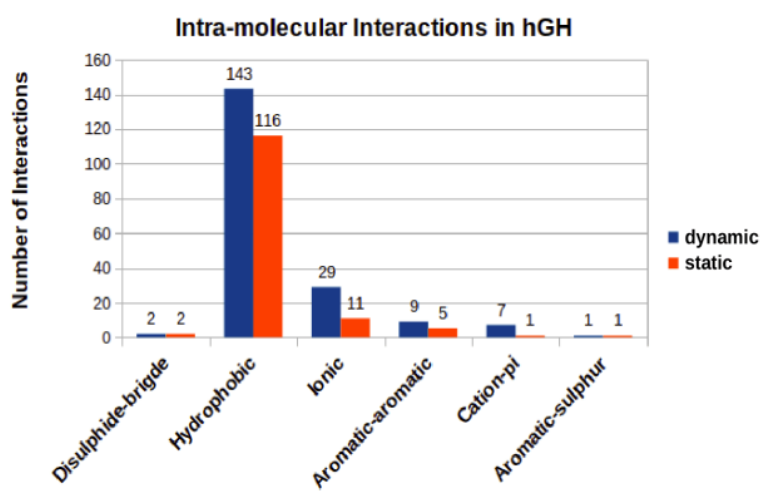

Fig. 6: Bar diagram showing different intra-molecular interactions in hGH molecule.

The bar diagram shown in Fig. 6 summarizes the various intra-molecular interactions present in hGH molecule in both structures i.e., static and dynamic conditions. The comparison diagram shows that hydrophobic interactions have the maximum binding sites and aromatic-sulphur has minimum binding sites.

Table 1: Ionic Interactions within $6 \AA$ (in static structure)

\begin{tabular}{|c|c|c|c|}
\hline Position & Residue & Position & Residue \\
\hline 16 & ARG & 107 & ASP \\
\hline 16 & ARG & 116 & ASP \\
\hline 18 & HIS & 174 & GLU \\
\hline 21 & HIS & 174 & GLU \\
\hline 32 & GLU & 41 & LYS \\
\hline 94 & ARG & 109 & ASP \\
\hline 115 & LYS & 118 & GLU \\
\hline 145 & LYS & 169 & ASP \\
\hline 154 & ASP & 158 & LYS \\
\hline 167 & ARG & 171 & ASP \\
\hline 171 & ASP & 172 & LYS \\
\hline
\end{tabular}

Table 2: Ionic Interactions within $6 \AA$ (structure in dynamic)

\begin{tabular}{|c|c|c|c|}
\hline Position & Residue & Position & Residue \\
\hline 16 & ARG & 116 & ASP \\
\hline 19 & ARG & 107 & ASP \\
\hline 19 & $\mathrm{ARG}$ & 26 & ASP \\
\hline 26 & ASP & 29 & GLU \\
\hline 29 & GLU & 30 & GLU \\
\hline 29 & GLU & 32 & GLU \\
\hline 32 & GLU & 33 & GLU \\
\hline 32 & GLU & 41 & LYS \\
\hline 33 & GLU & 41 & LYS \\
\hline 38 & LYS & 39 & GLU \\
\hline 64 & ARG & 65 & GLU \\
\hline 94 & ARG & 109 & ASP \\
\hline 94 & $\mathrm{ARG}$ & 112 & ASP \\
\hline 109 & ASP & 112 & ASP \\
\hline 112 & ASP & 115 & LYS \\
\hline 115 & LYS & 116 & ASP \\
\hline 115 & LYS & 118 & GLU \\
\hline 116 & ASP & 119 & GLU \\
\hline 118 & GLU & 119 & GLU \\
\hline 129 & GLU & 130 & ASP \\
\hline 129 & GLU & 134 & ARG \\
\hline 130 & ASP & 134 & ARG \\
\hline 153 & ASP & 154 & ASP \\
\hline 168 & LYS & 169 & ASP \\
\hline 168 & LYS & 171 & ASP \\
\hline 169 & ASP & 172 & LYS \\
\hline 171 & ASP & 172 & LYS \\
\hline 171 & ASP & 174 & GLU \\
\hline 174 & GLU & 178 & ARG \\
\hline
\end{tabular}

The aromatic-aromatic interactions also play important role in the structural stability of proteins. These interactions are significant within 4.5 to $7 \AA$ range between aromatic residues. In human growth hormone protein, we have detected 9 and 5 aromatic-aromatic interactions for the dynamic and static structures respectively. These interactions are presented in tables 3 and 4 . Moreover, there is an aromatic-sulphur interaction within $5.3 \AA$ present between PHE166 and MET170 residues in both 
structures. In static structure, the distance between the centroid and sulphur was observed $4.66 \AA$ and the angle was $72.7^{\circ}$, whereas in dynamic structure the distance between the centroid and sulphur is $5.01 \AA$ and the angle is $44.6^{\circ}$.

The cation-pi interactions occur between the cations of the side chains of arginine or lysine residues with the polarizable pi electron of the aromatic ring. These interactions are distance dependent interactions. Here, we have taken the cut off distance of $6 \AA$. In static structure, there is only one cation-pi interaction present between the residues LYS41 and TYR164 within the distance of $5.8 \AA$ and at an angle of $119.7^{\circ}$. However, in case of structure obtained from dynamics, we observed 7 cation-pi interactions between the ARG and LYS residues with the aromatic residues PHE and TRP as shown in table 5 .

Table 3: Aromatic-Aromatic Interactions within 4.5 and $7 \AA$ (in static structure).

\begin{tabular}{|c|c|c|c|c|c|}
\hline Position & Residue & Position & Residue & D(Centroid-Centroid) & Dihedral Angle \\
\hline 25 & PHE & 28 & TYR & 6.93 & 122.2 \\
\hline 31 & PHE & 35 & TYR & 4.73 & 132.8 \\
\hline 54 & PHE & 143 & TYR & 6.05 & 25.35 \\
\hline 86 & TRP & 166 & PHE & 4.93 & 50.11 \\
\hline 160 & TYR & 164 & TYR & 6.24 & 95.19 \\
\hline
\end{tabular}

Table 4: Aromatic-Aromatic Interactions within 4.5 and $7 \AA$ (structure in dynamic).

\begin{tabular}{|c|c|c|c|c|c|}
\hline Position & Residue & Position & Residue & D(Centroid-Centroid) & Dihedral Angle \\
\hline 25 & PHE & 28 & TYR & 6.14 & 144.03 \\
\hline 28 & TYR & 160 & TYR & 4.74 & 8.09 \\
\hline 28 & TYR & 164 & TYR & 6.07 & 31.76 \\
\hline 31 & PHE & 35 & TYR & 5 & 118.96 \\
\hline 54 & PHE & 143 & TYR & 6.74 & 50.35 \\
\hline 86 & TRP & 166 & PHE & 5.63 & 51.6 \\
\hline 86 & TRP & 97 & PHE & 6.4 & 22.9 \\
\hline 97 & PHE & 146 & PHE & 5.18 & 123.48 \\
\hline 97 & PHE & 166 & PHE & 4.79 & 37.53 \\
\hline
\end{tabular}

Table 5: Cation-Pi Interactions within 6 Å (structure in dynamic)

\begin{tabular}{|c|c|c|c|c|c|}
\hline Position & Residue & Position & Residue & D (Cation-Pi) & Angle \\
\hline 1 & PHE & 16 & ARG & 5.15 & 108.14 \\
\hline 1 & PHE & 8 & ARG & 5.32 & 119.02 \\
\hline 25 & PHE & 167 & ARG & 3.9 & 13.26 \\
\hline 42 & TYR & 41 & LYS & 4.54 & 141.61 \\
\hline 111 & TYR & 94 & ARG & 3.98 & 156.72 \\
\hline 139 & PHE & 77 & ARG & 3.93 & 149.17 \\
\hline 191 & PHE & 64 & ARG & 4.45 & 47.09 \\
\hline
\end{tabular}

\section{CONCLUSIONS}

We have carried out the molecular dynamics (MD) simulations in order to identify the intra-molecular contacts and their contributions in forming the stable structure of human growth hormone. The MD run was propagated for 100 ns simulation 
under NVT condition at body temperature $310 \mathrm{~K}$. We have studied bonded and non-bonded interactions which contribute in the formation of stable structure in aqueous environment. Many nonbonded contacts are compared in static and dynamic conditions.

The decreasing nature of solvent accessible surface area (SASA) depicts the hydrophobic nature of hGH which makes the structure more stable in water. We have compared the various intramolecular interactions namely disulphide bonds, hydrophobic, ionic, aromatic-aromatic, aromaticsulphur, cation-pi interactions between the structures from dynamics and statics. In the structure taken from the simulation at the condition of minimum SASA, we have found hydrophobic, ionic, cation-pi, aromatic-aromatic interactions significantly higher than that of static structure. The hydrogen bonding is observed strongly contributing in intramolecular binding. In addition, the estimation of bonded and non-bonded energy profiles show that electrostatic interactions have higher contributions in non-bonded condition. Similarly, the harmonic angle and dihedral angle have almost equal contribution in the conformation of the molecule. Bond energy contribution has also significant role but smaller than that of angle and dihedral.

\section{ACKNOWLEDGEMENTS}

RPK and SPK acknowledge the partial financial support from the Nepal Academy of Science and Technology (NAST). NPA acknowledges the UGC Award No. CRG-73/74-S\&T-01. We acknowledge the computing facilities of Supercomputer Center Kathmandu University, which was established with equipment donated by CERN and the Arkansas High Performance Computing Center which is funded through multiple National Science Foundation grants and Arkansas Economic Development Commission.

\section{REFERENCES}

[1] Fayter, D.; Nixon, J.; Hartley, S.; Rithalia, A.; Butler, G.; Rudolf, M.; Glasziou, P.; Bland, M.; Stirk, L.; and Westwood, M. Effectiveness and cost-effectiveness of height-screening programmes during the primary school years: a systematic review, Archives of disease in childhood, 93: 278-284 (2008).

[2] Greenwood, F.; and Landon, J. Growth hormone secretion in response to stress in man, Nature, 210: 540-541 (1966).
[3] Aloj, S.; and Edelhoch, H. The molecular properties of human growth hormone, Journal of Biological Chemistry, 247: 1146-1152 (1972).

[4] Kohler, M.; Püschel, K.; Sakharov, D.; Tonevitskiy, A.; Schänzer, W.; and Thevis, M. Detection of recombinant growth hormone in human plasma by a 2-D PAGE method, Electrophoresis, 29: 4495-4502 (2008).

[5] Li, C. H. Human growth hormone: 1974-1981, Molecular and cellular biochemistry, 46: 31-41 (1982).

[6] Yi, S.; Bernat, B.; Pál, G.; Kossiakoff, A.; and Li, W.-H. Functional promiscuity of squirrel monkey growth hormone receptor toward both primate and nonprimate growth hormones, Molecular biology and evolution, 19: 1083-1092 (2002).

[7] Koirala, R. P.; Pradhan, S.; and Aryal, S. K. Ultrasonic Measurement of Kidney Length in Nepalese People. Journal of Nepal Physical Society, 4(1): 49-53 (2017).

[8] Wells, J. A.; Cunningram, B. C.; Fuh, G.; Lowman, H. B.; Ultsch, M.; Devos, A. M.; Bass, S. H.; Mulkerrin, M. G. The molecular basis for growth hormone-receptor interactions, In Recent Progress in Hormone Research, 253-275, Elsevier (1993).

[9] Meiering, E. M.; Serrano, L.; and Fersht, A. R. Effect of active site residues in barnase on activity and stability, Journal of molecular biology, 225: 585-589 (1992).

[10] Schulga, A. A.; Makarov, A. A.; Levichkin, I. V.; Belousova, Y. V.; Lobachov, V. M.; Protasevich, I. I.; Pace, C. N.; and Kirpichnikov, M. P. Increased stability of human growth hormone with reduced lactogenic potency, FEBS letters, 528: 257-260 (2002).

[11] Burley, S.; and Petsko, G. A. Aromatic-aromatic interaction: a mechanism of protein structure stabilization, Science, 229: 23-28 (1985).

[12] Bhattacharyya, R.; Samanta, U.; and Chakrabarti, P. Aromatic-aromatic interactions in and around $\alpha$-helices, Protein engineering, 15: 91-100 (2002).

[13] Dahiyat, B. I.; and Mayo, S. L. Protein design automation, Protein Science, 5: 895-903 (1996).

[14] Kellis, J. T.; Nyberg, K.; and Fersht, A. R. Contribution of hydrophobic interactions to protein stability, Nature, 333: 784-786 (1988).

[15] Berman, H. M.; Westbrook, J.; Feng, Z.; Gilliland, G.; Bhat, T. N.; Weissig, H.; Shindyalov, I. N.; and Bourne, P. E. The protein data bank, Nucleic acids research, 28: 235-242 (2000).

[16] Lee, J.; Cheng, X.; Swails, J. M.; Yeom, M. S.; Eastman, P. K.; Lemkul, J. A.; Wei, S.; Buckner, J.; Jeong, J. C.; Qi, Y.; Jo, S.; Pande, V. S.; Case, D. A.; Brooks, C. L. 3rd, MacKerell, A. D.; Jr., Klauda, J. B.; and Im, W. CHARMM-GUI Input Generator for NAMD, 
GROMACS, AMBER, OpenMM, and CHARMM/OpenMM Simulations Using the CHARMM36 Additive Force Field, $J$ Chem Theory Comput, 12: 405-413 (2016).

[17] Huang, J.; Rauscher, S.; Nawrocki, G.; Ran, T.; Feig, M.; de Groot, B. L.; Grubmuller, H.; and MacKerell, A. D., Jr. CHARMM36m: an improved force field for folded and intrinsically disordered proteins, Nat Methods, 14: 71-73 (2017).

[18] Phillips, J. C.; Braun, R.; Wang, W.; Gumbart, J.; Tajkhorshid, E.; Villa, E.; Chipot, C.; Skeel, R. D.; Kale, L.; and Schulten, K. Scalable molecular dynamics with NAMD, J Comput Chem, 26: 1781-1802 (2005).

[19] Koirala, R. P.; Bhusal, H. P.; Khanal, S. P.; and Adhikari, N. P. Effect of temperature on transport properties of cysteine in water, AIP Advances, 10: 025122 (2020).

[20] Ramachandran, S.; Kota, P.; Ding, F.; and Dokholyan, N. V. Automated minimization of steric clashes in protein structures, Proteins, 79: 261-270 (2011).

[21] Pantha, N.; Chauhan, B.; Sharma, P.; and Adhikari, N. P. Tuning Structural and Electronic Properties of Phosphorene with Vacancies. Journal of Nepal Physical Society, 6(1): 7-15 (2020).
[22] Walton, E. B.; and Vanvliet, K. J. Equilibration of experimentally determined protein structures for molecular dynamics simulation, Phys Rev E Stat Nonlin Soft Matter Phys, 74: 061901 (2006).

[23] Humphrey, W.; Dalke, A.; and Schulten, K. VMD: visual molecular dynamics, J Mol Graph, 14: 33-38, 27-38 (1996).

[24] Tina, K.; Bhadra, R.; and Srinivasan, N. PIC: protein interactions calculator, Nucleic acids research, 35: W473-W476 (2007).

[25] Neopane, S.; and Pantha, N. First-Principles Study of van der Waals Interactions between Halogen Molecules (Cl2 and I2). Journal of Nepal Physical Society, 5(1): 19-23 (2019).

[26] Thakuria, R.; Sarma, B.; and Nangia, A. 7.03 Hydrogen Bonding in Molecular Crystals, Comprehensive Supramolecular Chemistry, II: 25-48 (2017).

[27] DiStasio Jr, R. A.; Gobre, V. V.; and Tkatchenko, A. Many-body van der Waals interactions in molecules and condensed matter, Journal of Physics: Condensed Matter, 26: 213202 (2014).

[28] Yunta, M. It is important to compute intramolecular hydrogen bonding in drug design, American Journal of Modeling and Optimization, 5: 24-57 (2017).

[29] Hubbard, R. E.; and Haider, M. K. Hydrogen bonds in proteins: role and strength, $e L S$ (2010). 